\title{
COMMISSION 20: POSITIONS AND MOTIONS OF MINOR PLANETS, COMETS AND SATELLITES \\ (POSITIONS ET MOUVEMENTS DES PETITES PLANETES, DES COMETES ET DES SATELLITES)
}

PRESIDENT: R.M.WEST

VICE-PRESIDENT: A.CARUSI

Organizing CommitTeE: K.Aksnes, J.-E.Arlot, Y.Kozai, L.Kresák, B.G.Marsden, H.RickMan, V.A.Shor, L.H.Wasserman, D.K.YeOmans, J.-X.ZhanG

\section{Introduction}

The past triennium was characterized by important advances in several directions within the broad field of this Commission. Among the most exciting events were the Phobos 2 and Voyager 2 missions to Mars/Phobos and Neptune/Triton; their navigation was critically dependent on precise space- and ground-based observations as well as refined theoretical models of the motions in the solar system. Among many other achievements, the secular acceleration of Phobos was accurately measured and the dynamical analysis of the ring-like arcs at Neptune has just begun.

At the end of June 1990, 4508 minor planets had been numbered, an increase of no less than 878 since the last triennium. This is due to the impressive rate of successful identifications at the Minor Planet Center and elsewhere, facilitated by efficient distribution of the newest data in the Minor Planet Circulars, now also in computer-readable form. Older observations are being critically studied; a reliable and complete data base with all positional observations will soon become available. $90 \%$ of the orbits in the annual Minor Planet Ephemerides from the Institute for Theoretical Astronomy (Leningrad) are now good to 4 arcsec, i.e. sufficiently accurate for automatic object acquisition with most modern observational equipment.

There were a record 79 comet discoveries/recoveries during the triennium. Improved instrumentation made it possible to study comets at ever larger distances, and the non-gravitational forces and dynamical chaos were central theoretical subjects. New work was done on the evolution of cometary orbits, including the long-standing question of the origin. The structure of the cometary reservoirs in the outer solar system is not yet well established and various mechanisms have been studied regarding their ability to supply new objects at the observed rate.

Occultation events attract an increasing number of observers; this has resulted in a number of very good new determinations of minor planet diameters. The unique Pluto/Charon eclipses have given the first indications about surface structures on these distant objects.

In addition to the four Working Groups of Commission 20 (Minor Planets, Comets, Satellites, Occultations), whose chairmen have compiled the corresponding sections of this report, the Satellite Nomenclature Liaison Committee (Chairman: K.Aksnes), the Minor Planet Names Committee, the Comet Nomenclature Committee and the Standing Committee to Oversee Publication of Photometric Data continued their work. For the latter, E.Tedesco has undertaken a revision of the $\mathrm{H}$ and $\mathrm{G}$ magnitude parameters of the numbered minor planets. L.Schmadel, who took over from E.Bowell the chairmanship of the new Working Group on the Origin of Minor Planet Names, reports that work is well underway on a new dictionary of names and that only 160 names have unknown origins, while 59 more are uncertain or questionable. The System Transition Committee (Chairman: D.K.Yeomans) presented its recommendations for the B1950 to J2000 transition, now planned for 1992 .

Many papers of general interest can be found in "Asteroids II" (A II) (Tucson, 1989); "Asteroids, Comets, Meteors III" (ACM III) (Uppsala, 1990); in the Proc. of Course 98 of the Enrico Fermi Int. School of Physics "The Evolution of the Small Bodies of the Solar System" (44.012.041); IAU Coll. 96 "The Few Body Problem" (045.012.051); IAU Coll. 98 "Stargazers" (49.012.008); IAU Coll. 116 "Comets in the Post Halley Era" (Kluwer, 1990); "Orbital Dynamics of Natural and Artificial Objects" (ODNAO) (Obs. Nacional, Rio de Janeiro, 1990). 
It is a sad duty to record the deaths of Willy Strobel, Maciej Bielicki, Leland E. Cunningham, Edgar Everhart III and Jack Bennett. With them, the Commission has lost some of its most active and venerable members.

\section{Minor Planets (B.G. Marsden)}

\section{II.1 General}

Astrometric and orbital work on minor planets has continued at an ever-increasing rate, the most visible evidence for this being monthly batches of Minor Planet Circulars (MPC) that have consistently run to well over 100 pages (sometimes almost 200) and annual volumes of Efemeridy Malykh Planet (EMP) that increased from 366 pages (1988 edition) to 447 (1991).

Since 1978, the $M P C$ s, which principally contain the observations, orbital elements, ephemerides for unnumbered and newly numbered minor planets and new assignments of names, have been issued by the Minor Planet Center at the Smithsonian Astrophysical Observatory, Cambridge, Mass., under the direction of Marsden. Bardwell, who has been associated with the Minor Planet Center since 1958 , officially retired at the end of 1989 , although he has continued to work at a reduced level. During the early 1960s he became the first person to attempt to automate searches for identifications of minor planets, and his success soon began to quicken the pace at which new minor planets were numbered. Green and Nakano also continued to participate in the work of the Minor Planet Center; the latter, who has in recent years been the leader in the development of the autornated identification searches begun by Bardwell, returned to Japan in mid-1990 after a four-year stay in Cambridge but continues to serve as the liaison between the Minor Planet Center and the now rather large group of Japanese amateur astronomers involved with both observations and orbits. Early in $1990 \mathrm{G}$. Williams, among whose particular interests has been the correct identification of observations of numbered minor planets and the detective work involved in attending to the numerous longstanding errors in the observation files, came from England to be a consultant at the Minor Planet Center, and later in the year he was appointed successor to Bardwell as Associate Director.

Also by longstanding arrangement, the $E M P$, containing the ephemerides of the established numbered minor planets and their orbital elements updated to a current epoch of osculation, has been published by the Institute for Theoretical Astronomy (ITA), Leningrad, with Batrakov as editor-in-chief and Shor as deputy. Shor (ACM III 179) discussed the likely future evolution of the $E M P$ into a computer diskette form. Ashkova, who had been extensively involved in the preparation of the EMP since she joined the ITA in 1954, retired in 1987. Izvekov, another extensive EMP contributor on the ITA staff since 1951 and one of the first in the U.S.S.R. to use electronic computers for the computation of orbits and ephemerides, retired in 1988. Others involved in the computation of data for the EMP were Chernetenko, Filenko, Sumzina, Vasil'kova and Vinogradova. Through 1987 Syromyatnikov and Pavlova assisted with the preparation of the EMP, and typesetting programmes were written by Filippova and Glejbman. Filippova, Vinogradova and Kastel' have also computed preliminary orbits for new objects.

Since 1986 the $M P C$ s have been issued both in printed and diskette form; the availability of the latter for updating computer files has meant that revised tapes with the complete set of observations are issued less frequently than before. A tape version was issued by the Minor Planet Center in 1989, and the number of observations of minor planets on it (337039 of numbered objects, 173232 of unnumbered) represents a $35 \%$ increase over its 1986 predecessor.

The Minor Planet Center issued in 1989 a new edition of the "Catalogue of Orbits of Unnumbered Minor Planets" with 6965 orbits listed in the printed edition. The number of multipleopposition orbits, 1973, compares with 855 (1986 edition) and only 211 (1982). The diskette edition of the 1989 catalogue, with a total of 11706 orbits, includes the Palomar-Leiden orbits, as well as those from the Palomar-Leiden Trojan surveys described in II.2. For convenience, the orbit diskettes also include the orbits of the numbered minor planets for the standard epoch 1990 Nov. 5.0. Most of these are the ITA computations given in EMP 1990, although the number of objects was extended from 3936 to 4295 , and improved data were also available for many of the older objects. The orbits updated at ITA on an annual basis are also available from the 
Stellar Data Center (CDS), Strasbourg. Orbits of numbered, multiple-opposition and recent single-opposition minor planets can also be accessed individually through the Computer Service the Minor Planet Center operates with the Central Bureau for Astronomical Telegrams (CBAT), and there is in addition a facility for computing ephemerides.

Beginning with EMP 1989 some improvements were made in the published form of the ephemerides, although there were still eight dates at ten-day intervals in each ephemeris and twelve ephemerides per page. Positions are now given to 0.1 arcmin, rather than 1 arcmin, and the geocentric and heliocentric distances, the magnitude and the phase angle, are now given for each date. Variations are no longer given, but a header line includes an indication of the expected accuracy of each ephemeris. Except for some of the objects on the critical list, it is very unlikely that an ephemeris will be in error by more than about 10 arcsec. A plot by Bowell, Chernykh and Marsden ( $A$ II 21 ) indicates that fully $90 \%$ of the orbits are capable of giving ephemerides that are good now to 4 arcsec, and $50 \%$ are good to 2 arcsec.

This reference also introduced the request that discoverers of new minor planets report to the Minor Planet Center accurate positions of these objects on two nights. In addition, except for unusual objects in the vicinity of the earth, it has been requested that observers report at that time all the observations made of a particular object on those two nights (preferably two on each), and that all other observations made of it at the same dark run (preferably on two more nights separated from the others to give a total arc of ten days or more) should be reported in at most one more communication. Observations on three (or more) nights covering no more than four or five days generally represent a wasted effort. It is of course highly desirable that observers be sure to link correctly the observations made on the two nights, and it is far preferable that they concentrate on this (and making the measurements accurately, not to mention timing their exposures properly) than that they actually try to identify the objects. Although timing errors are still surprisingly common, general compliance with these requests has, as anticipated, continued to improve the efficiency of operation of the Minor Planet Center, allowing in particular the more-or-less routine immediate recognition of even quite sophisticated identifications. This in turn allows the Minor Planet Center to advise observers whether further observations of an object are in fact needed, and by concentrating on obtaining good arcs for new objects that cannot be identified they enable the computation of long-arc orbits that can appropriately be used for searches at other oppositions. There has therefore been a reduction in the number of short-arc, indeterminate orbits in which some assumptions have to be made in order to obtain a result that merely looks reasonable. It is sometimes inevitable that observations can be made on only one night, but the processing of these observations by the Minor Planet Center is now being delayed in the hope that the objects are reported on multiple nights by others, who then get credit for the discoveries. It is anticipated that these delays will become longer in the future, and it is earnestly hoped that the number of "one-night stands" will continue to decrease.

As of June 1990 there were 4508 numbered minor planets, an increase of 878 during the triennium. Although this increase is impressive, the even more dramatic increase noted above in the number of unnumbered multiple-opposition orbits suggests that many more minor planets could perhaps have been numbered. The problem is that the standard of accuracy of the orbits of the older numbered minor planets is now noticeably greater than would be achieved for minor planets newly numbered, according to convention, as soon as there are observations at three, perhaps four oppositions. While a few minor planets are now being numbered when there is good coverage at each of three oppositions (and earth-approaching objects are still often done from only two oppositions), the Minor Planet Center now tends to refrain from numbering objects at as many as four oppositions unless there is an arc of a month at two of those oppositions. When a multiple-opposition object is recovered (on, say, neighbouring nights at the dark run preceding the next opposition), it seems not much to ask that a follow-up be made a month later, and the resulting measurements do have a noticeable effect on the orbital accuracy.

\section{II.2 Observations and Orbits}

Photographic observing programmes that include an emphasis on the discovery and followup of new minor planets have continued at Brorfelde (Jensen), Caussols (Maury and others), 
El Leoncito (Sanguin), Kiso (Nakamura and others), Klět (Mrkos and Vávrová), Kvistaberg (Oja), La Silla (Debehogne, Elst and others), Mount John (Gilmore and Kilmartin), Nauchnyj (Chernykh and others), Palomar (Helin, Shoemaker and others), Piwnice (Antal), Siding Spring (McNaught and others), Smolyan (Elst, Shkodrov and others), Tautenburg (Börngen), Victoria (Tatum and Balam) and Zimmerwald (Wild). The photographic programme carried out with the Pluto Telescope in Flagstaff by Bowell and his colleagues was essentially terminated; this team now puts its effort into observations with larger Schmidt telescopes at Palomar, Siding Spring and Cerro Tololo, and CCD observations in Flagstaff. The programme conducted with the Nanking astrograph under the direction of Zhang was also phased out in favour of one at the Peking Observatory's Xinglong Station, as well as a new CCD programme at Yunnan. Gibson continued his CCD follow-up programme at Palomar. The Oak Ridge programme (McCrosky and Shao) was converted from photographic to CCD with no break in continuity. The University of Arizona's Spacewatch programme (Gehrels, Scotti and others) was out of action for much of the triennium during the conversion from a $512 \times 320$ to a $2048 \times 2048$ CCD. Rajamohan's programme at Kavalur was converted from photographic to a CCD programme along Spacewatch lines. Tholen has obtained some very timely and useful follow-up astrometry with the encoders on telescopes at Mauna Kea. New photographic programmes were undertaken at Haute-Provence (Elst and Sause) and Merida (Geffert and others). More than three dozen amateur astronomers in Japan participated in an exceptionally active search programme coordinated by Nakano. There were also several discoveries by amateur astronomers in Italy (Baur and Colombini) and England (Manning), and follow-up by others in these countries as well as in France (Chanal), Australia (Herald) and U.S.A. (Handley).

Series of observations with an emphasis on numbered minor planets include the photographic programmes at Barcelona (Codina), Chorzow (Włodarczyk), Lick (Klemola), Perth (Candy), Poznań (Hurnik), San Fernando (Quijano), Valencia (Lopez), Wroctaw (Bem), Yebes (MartínPintado), by amateur astronomers principally in West Germany (Bonk, Bressler, Kretlow and Seiler) and Italy (Lai and Sicoli), as well as by professional groups at several observatories in the U.S.S.R. in connection with the ITA programme on 20 selected objects. Quijano specifically reports that 435 positions have been measured on 145 plates exposed with the Carte du Ciel astrograph for the ITA project and the 63 objects for the Hipparcos project. Series of observations for the Hipparcos project were also made with the automatic meridian circles at Bordeaux (Rapaport) and La Palma (Morrison), and new orbits for the Hipparcos objects have been determined by Bec-Borsenberger (46.098.042) and Calaf (46.098.043).

One more lost numbered minor planet was identified during the triennium, leaving now only (719) Albert and (878) Mildred to be found. (724) Hapag, previously observed only over a one-month arc by Palisa at Vienna in 1911, was identified by Nakano with $1988 \mathrm{VG}_{2}$, an object observed over a seven-day arc by Japanese amateurs Hioki and Kawasato. Following this, West and McNaught quickly identified further images on copies of Sky Survey plates taken at Palomar and Siding Spring in 1953, 1955, 1983, 1984 and 1987; with a total of eight oppositions the object could be removed from the critical list. Kristensen and West (50.098.011) investigated the available data on (719), including a re-reduction of the Copenhagen and Heidelberg observations.

Late in 1987 the extensive collection of Indiana plates of minor planets was moved to Flagstaff. Measurements from them are now being made systematically by Bus, Skiff and others under Bowell's general direction, and also of the old Lowell plates. More than in the recent past, much of the emphasis of these old measurements has been on numbered minor planets, and Bowell has also been actively involved in redetermining their orbits, as have Goffin (Mortsel) and the staffs of ITA and the Minor Planet Center.

In the report covering the 1981-84 triennium, it was remarked that the time-consuming process of deleting, correcting or otherwise identifying observations of numbered minor planets showing large residuals had been completed from the most recently numbered minor planets back to (901). Although some work was subsequently done at the Minor Planet Center on the further backward extension to this, progress was slow, and nothing was close to the stage where amendments were ready for implementation in the observation files and publication in the MPCs. During his consultancy at the Minor Planet Center, G. Williams gave higher priority to this work and by June 1990 had implemented and arranged the publication of the outcome back to (601). 
In the course of reorganizing this work he revised and greatly extended an earlier preparation by Scholl of sets of osculating elements of the numbered minor planets at 200-day intervals back to 1899 and was at the same time able to make some 400 more identifications of provisionally designated minor planets with numbered objects. His surprisingly large addendum to the 1982 survey by Schmadel included unexpected results such as $(6)=1947 \mathrm{JB},(14)=\mathrm{A} 906 \mathrm{QC},(34)=$ $1965 \mathrm{JL},(36)=\mathrm{A} 901 \mathrm{SB},(433)=1956 \mathrm{PC}$ and $(1269)=\mathrm{A} 902 \mathrm{EA}=\mathrm{A} 906 \mathrm{SA}=\mathrm{A} 907 \mathrm{WB}=$ A918 EG $=1926 \mathrm{GC}_{1}$. When it becarne desirable to determine new orbits he also made an effort to add to the observation files many observations preceding the nominal 1939 date back to which the Minor Planet Center's files are supposedly complete; in many instances this involved an automatic re-reduction of the old micrometric observations. At the current rate of progress, the first pass at the file correction should be completed by the end of 1990 . A second pass through the whole set of numbered minor planets is a simpler proposition and should be completed prior to the transformation of the observations from the FK4/1950.0 system to FK5/J2000.0 planned for the end of 1991.

As already noted, fairly extensive identification searches are now made quite routinely for new discoveries, particularly at the Minor Planet Center and by several Japanese amateurs. The Canadian amateur Lowe has again contributed several identifications involving cases where there were observations on no more than two nights at any opposition. Although the identifiers usually complete the work by computing final orbits, some final orbits have also been computed by Balam, Bowell, Landgraf and Schmadel, as well as by the U.S. amateurs Rogers and Townsend.

During the triennium as many as 27 new Apollo and Aten objects were discovered. Among them was $1989 \mathrm{FC}$, which made a record close approach to within $0.0046 \mathrm{AU}$ of the earth some eight days before discovery, and the approaches of $1989 \mathrm{AC}$ within $0.02 \mathrm{AU}$ of the earth in 1992 and to $0.01 \mathrm{AU}$ in 2004 will each break the record miss distance predicted for any celestial object other than the moon. By the end of the triennium five of the 27 (incl. 1989 AC) had in fact been numbered, and three more (incl. 1989 FC) had been identified at a second opposition. Ten earlier Apollo and Aten objects were also given permanent numbers during 1987-1990.

Van Houten and van Houten-Groeneveld report that the Third Palomar-Leiden Trojan Survey (T-3) was completed (50.098.042); preliminary orbits from the 1977 observations were published (MPC 12536-12560). The T-2 survey of 1973 was also completed, a paper being submitted for publication and preliminary orbits being published (MPC 14907-14930). The T-2 survey yielded orbits for 1504 objects, of which 55 are Trojans, 12 are Hildas and 5 are Hungarias. A preliminary discussion of the differences between the $\mathrm{L}_{4}$ and the $\mathrm{L}_{5}$ Trojans indicated that the former are concentrated more strongly to the ecliptic than the latter and that they are about twice as numerous to a brightness limit of 20.0 (photogr.) at opposition. Work is well underway on the T-1 survey, a total of 53 plates involving four fields close to $\mathrm{L}_{5}$, photographed at opposition in March 1971, a blink pair in April and two plates on two nights in May. The measurement of the positions (van Houten-Groeneveld) and magnitudes (Wisse-Schouten) is practically complete, and the orbits and necessary ephemerides are mostly being calculated at the Minor Planet Center by Green.

As many as 21 Trojans were permanently numbered during the triennium, augmenting the previously numbered Trojans by precisely $50 \%$. Eight of the new numberings involved discoveries made in the course of new searches, notably by Shoemaker, who with Wolfe ( $A I I 487)$ notes that the total population of Trojans larger than $15 \mathrm{~km}$ in diameter is roughly half that estimated for main-belt objects; they also suggest the presence of five and possibly as many as eight collisional groups around $\mathrm{L}_{4}$. During 1987-1990 there were as many as 61 other multiple-opposition Trojan discoveries, as well as 38 new Trojans observed at a single opposition.

\section{II.3 Theoretical Investigations}

There continues to be interest in developing efficient ways of identifying all the observations of a particular object at its discovery opposition. Following a discussion ( $A A I I 21)$ of generalization of the use of Väisälä orbits to nonapsidal cases, Bowell et al. (ACM III 19) suggested that observed motion vectors at opposition could be transformed rather unequivocally into semimajor axis and inclination, while Kristensen (Astr. Nachr. 311, 133, 1990) illustrated how one could 
use even a single position to derive the time of opposition and the minimum inclination.

Kresák and Klačka (49.098.048) discussed selection effects in the sample of numbered minor planets. They explained the well-known peak of discoveries during August-November (and a smaller peak during February-March) as due to a combination of the effects of the alignment of the orbital axes with that of Jupiter, the departure of the opposition point from the confusing sky background near the galactic equator and the altitude of the opposition point and length of night at the north temperate latitudes of the vast majority of the participating observatories.

J. Williams published a tabulation of proper elements and family membership for the first 3040 minor planets ( $A$ II 1034), and with Hierath he determined and discussed proper elements and family memberships for the Palomar-Leiden survey (44.098.060). Wolfe determined proper elements for the Palomar-Leiden and UCAS orbits and identified possible new families of faint minor planets, including one family of Mars crossers that would presumably be of quite young age. Reviews on the families were also given by Kozai (49.098.141, 50.098.001), Zappalà (49.098.142) and Chapman et al. ( $A$ II 386). Paolicchi and Zappalà (46.098.087) discussed problems concerning the interpretation of families as the result of catastrophic processes. Farinella et al. (49.098.012) reassessed Brouwer's work on groups within families and concluded that it may have validity, specifically with regard to the idea that a subset of the Eos family and the IRAS dust-band detection may have resulted from a collision only $10^{6}$ years ago. Sykes (Icarus $\mathbf{8 5}, 267,1990$ ) investigated the relationship between the families and these dust bands in more detail.

Knežević and Milani ( $A$ II 1073) also published a new set of proper elements, while Knežević et al. (45.098.016) compared short-period perturbations resulting from the Yuasa theory with numerical integrations, finding that in most cases a simple and fast analytical procedure can provide data accurate enough for the reliable classification into families.

Schubart extended his earlier numerical studies on the long-period evolution of Hilda-type orbits. He considered real orbits of special interest, together with related cases at other resonances (46.098.129) and orbits corresponding to a statistical gap in the distribution of a proper parameter of eccentricity ( $A C M I I I$ 171). Continuing his earlier work on motion near first-order resonances in the planar restricted three-body problem, Ferraz-Mello (46.098.001) examined the high-eccentricity libration of the Hildas and the absence of apocentric librators and inner circulators. From the same precepts Gerasimov (46.098.096) established expressions for the maximum changes in eccentricity and the periods of variation for first-order commensurabilities. On the other hand, Sessin and Bressane (46.042.029) were unable to reproduce Schubart's quantitative results from the elliptic planar restricted problem. Šidlichovský $(45.098 .002,45.098 .056)$ extended Wisdom's method to the 5:2 resonance, establishing the possibility of transitions between modes of motion with high and low eccentricity and Lyapunov exponents in such cases of $10^{-4} /$ year and of the collisional removal of objects from the resonance when the eccentricity approaches 0.4. Numerical integrations by Hahn et al. (ACM III 95) confirmed the possibility that objects there can become Mars crossing, as did computations by Ipatov (46.098.097) in both the $5: 2$ and $3: 1$ cases. In numerical experiments involving the $2: 1,3: 1$ and $3: 2$ resonances, Zhang et al. (46.098.003) noted that the characteristics of ideal resonance disappear when the eccentricity is less than that of Jupiter. By analyzing the distribution of trajectories near the equilibrium points in the phase plane Liu and Liao (50.098.095) arrived at a preliminary explanation of the gaps and clusters. Hadjidemetriou (46.042.083) applied a simple algebraic mapping to study perturbations near the 3:1 resonance. Noting that the probability of collisions decreases with increasing eccentricity, Kazantsev and Sherbaum (50.098.007) concluded that collisions do not influence the formation of the Kirkwood gaps.

Orellana and Vucetich (46.098.002) analyzed Trojan motion in terms of the principle of equivalence, determining a Nordtvedt parameter of $0.0 \pm 0.5$ and a Sun/Saturn mass ratio of $3497.92 \pm 0.76$. Innanen and Mikkola (49.042.001) found that there exists the possibility of stable Trojans for all the giant planets and that even in the Saturnian case there can be stability for at least $10^{7}$ years. They also noted that, contrary to intuition, there is empirical evidence for the stability of Trojan motion for all the terrestrial planets on timescales of more than $10^{6}$ years, a result rather dramatically confirmed by the discovery of $1990 \mathrm{MB}$, which indeed appears to be the first known "Mars Trojan". 
Yoshikawa $(44.098 .087,46.098 .085)$ examined the $\nu_{6}$ secular resonance both numerically and analytically, finding that there should be large changes in the orbital eccentricities (up to 0.8) of minor planets nearby and that the argument of the secular resonance should librate about $0^{\circ}$ or $180^{\circ}$. He also made (49.098.005; ACM III 207) a comprehensive survey of 28 meanmotion resonances. Froeschlé and Scholl (46.098.126) numerically considered orbital evolution in the vicinity of $\nu_{5}, \nu_{16}$ and the 5:1 Jupiter mean-motion resonance. Reviews on mean-motion resonances and secular resonances were published by Froeschlé et al. ( $A$ II 827; ACM III 63) and Scholl et al. ( $A I I$ 845), respectively, and Nobili ( $A I I$ 862) reviewed the effect of resonances on the depletion of the outer part of the belt. Franklin et al. (49.098.059) readdressed the question of the stability of orbits between Jupiter and Saturn, finding that integrations over $10^{6}$ Jupiter revolutions eliminate the possibly stable bands noted previously. He and Zhang (Act.Astr.Sin. 31 , 27, 1990) have used Broucke's Symbolic Poisson Series Processor to prepare a computer programme for the automatic computation of the general perturbations on the Flora group. They note increased accuracy and efficiency over the Bohlin group method, especially near the 7:2 and 10:3 resonances, but that Strömberg's table is otherwise quite suitable for use for mean motions between 1000 and $1100 \mathrm{arcsec} /$ day. Fernández and Beaugé (45.098.059) generalized Wilkens' method to establish temporary librations of (49) Pales and two other objects about the Laplacian 1:3:2 commensurability with Jupiter and Saturn.

Yeomans, Ostro and Chodas (44.098.020, 46.098.081) assessed the extent to which radar observations can improve the accuracy of ephemerides near the earth and discussed the particular case of $1986 \mathrm{JK}(49.098 .055)$.

Hahn (45.098.129) prepared a database of observing conditions and close approaches of AtenApollo-Amor objects during the present century and with Lagerkvist (46.098.127) carried out numerical integrations of the orbits of 26 objects over an interval of $10^{5}$ years. Extensive longterm integrations carried out by Milani et al. (49.098.046), while obviously not agreeing in all details, confirm a general situation of chaotic motion.

Olsson-Steel (44.098.029, 44.098.084, 46.098.035; ACM III 159) related the apparently Jupiter-crossing Apollo object 5025 P-L to the Taurid meteoroid complex and 1987 SY to the $\delta$ Leonids and found the characteristic timescale of dynamical loss by ejection from the solar system to be about $10^{6}$ years. Wetherill $(44.098 .027,46.098 .021)$ identified distinct portions of the minor-planet belt as source regions for ordinary chondritic meteorites and differentiated stony meteorites and noted suggestive, but not compelling, evidence that some Apollos and Amors are inactive comets.

New determinations of the masses of (1) Ceres, (2) Pallas and (4) Vesta, $5.0 \pm 0.2,1.4 \pm 0.2$ and $1.5 \pm 0.3$ (in units of $10^{-10} \mathrm{M}_{\odot}$ ), respectively, have been determined by Standish and Hellings (50.098.010), principally from the highly accurate ranging data to the Viking landers on Mars; the value for Ceres is similar to one determined by Landgraf (45.098.009) from the perturbations on Pallas. Fedij (46.098.121) noted that the perturbations by the first four minor planets on others can amount to 0.8 arcsec over 30 years. Hoffmann ( $A I I 228$ ) reviewed mass determinations of minor planets and noted 15 mutual encounters of minor planets within $0.01 \mathrm{AU}$ during 19801988 , suggesting that the approach between (804) and (1002) might allow a mass determination for the former (49.098.047).

Chauvineau and Mignard (46.098.091, 50.098.004; ACM III 29; Icarus 83, 360, 1990) readdressed the matter of satellites of minor planets, indicating that their orbits could be stable. Any observational evidence for them remains extremely questionable; see also Weidenschilling et al. ( $A$ II 643).

\section{Comets (H. Rickman)}

\section{III.1 Discoveries, Recoveries and Astrometric Observations}

The past triennium saw the discoveries of 38 long-period (LP) and 13 short-period (SP) comets, and 28 previously known short-period comets were recovered. In 1987 and 1989, more comets were discovered or recovered than there are letters in the alphabet; this had never happened before, and a new convention was introduced, repeating the letters with subscript 1 . The record 
number in a single year is now 34 in 1989 with designations up to and including $1989 \mathrm{~h}_{1}$. Of the 51 discoveries, 10 were made on images from the Solar Maximum Mission (SMM) coronagraph, and the rest were Earth-based. As earlier in the 1980's, amateurs contributed about $1 / 3$ of these - almost exclusively from visual search programmes. Nearly all discoveries by professional astronomers were done using photographic plates or films. One of the 13 discovered SP comets had earlier received an asteroidal designation: $1986 \mathrm{TF}=1987$ XXXVI P/Parker-Hartley, (Nakano, IAUC 4752). Other such identifications by Nakano referred to comets $\mathrm{P} /$ Smirnova-Chernykh in late 1982, P/Reinmuth 1 in early 1988, and P/Whipple observed as asteroid 1925 QD at a prediscovery apparition (1926 VIII: $M P C$ 12626). Furthermore, (2060) Chiron showed anomalous brightening (Tholen et al., IAUC 4554) and later exhibited a faint coma (Meech and Belton, IAUC 4770).

As usual, there was a very clear predominance of Earth-based discoveries from the Northern hemisphere: 12 out of 15 visual discoveries and 19 out of 26 photographic ones. This reflects the fact that a majority of the well-equipped and experienced, visual comet hunters operate from North America and Japan. It is noteworthy, however, that Bradfield (Australia) made his 13th and 14th discoveries, and he now ranks fifth on the all-time list of comet discoverers. Among the photographic programmes, those conducted at Palomar have made outstanding contributions with 16 discoveries during the triennium (one shared with the Brorfelde Observatory, Denmark), and this alone causes the hemispherical asymmetry of photographic discoveries. 8 of the Palomar discoveries come from the search programme conducted by E.M. and C.S. Shoemaker with the 46-cm Schmidt telescope, and since her 17th discovery, C.S. Shoemaker now ranks third on the all-time list. The Planet-Crossing Asteroid Survey (PCAS) programme conducted by Helin scored 5 discoveries with the same telescope, 4 discoveries were made at Siding Spring with the UK Schmidt, and the Palomar Sky Survey II had 3 discoveries at the 1.2-m Schmidt. Descriptions of search programmes were given by Shoemaker and Shoemaker (46.002.075), Dow and Sykes (46.098.018), Helin et al. (Lun.Plan.Sci. XXI, 491, 1990), and Parker et al. (ACM III 413). Yeates (50.036.237) and Frank et al. (ABA 228, 522, 1990) described a search project aimed at detecting meter-sized mini-comets in the Earth's immediate surroundings - a kind of body recently claimed to exist in enormous numbers although this would seem rather unlikely.

Recoveries of SP comets were mainly done with CCD detectors rather than photographically (21 vs. 5 cases; 2 cases of both techniques used independently). This is a natural development due to larger-field CCD's and better ephemerides becoming available. At present the main contributions are by Gibson at Palomar (16 recoveries of which 7 were shared with other observatories) and the Spacewatch programme at Kitt Peak (Gehrels and Scotti; 8 recoveries of which 4 were shared with other observatories). In 20 cases the recovery circumstances can be compared with earlier ephemeris-aided recoveries, and more than half of these cases occurred at larger pre-perihelion heliocentric distances than ever before, e.g. P/Longmore (4.09 AU), $\mathrm{P} /$ Kopff (4.72 AU), P/Schwassmann-Wachmann 3 (2.24 AU) and P/Honda-Mrkos-Pajdušáková $(1.60 \mathrm{AU})$. The median heliocentric distance for the 25 pre-perihelion recoveries was $2.6 \mathrm{AU}$ with quartiles at 2.1 and $3.6 \mathrm{AU}$.

Seven comets had their third observed apparitions, thus allowing reliable determinations of nongravitational effects (cf. III.2). One of these cases was remarkable: 1989o P/Brorsen-Metcalf (recovered by Helin with the Palomar $46-\mathrm{cm}$ Schmidt), the brightest predictable cometary apparition until well into the 21st century. Even though this first ephemeris-aided recovery took place at a larger heliocentric distance $(1.51 \mathrm{AU})$ than the comet had ever been observed before, it still came unexpectedly late since the total magnitude was reported as bright as 9 within a week of recovery. This was due to an unusually large offset $(\Delta T=-15.6$ days) of the time of perihelion passage with respect to the predicted orbit, caused by the nongravitational force. Six comets were observed at their critical, second apparitions - the first apparitions having occurred in 1973-1984 and no apparitions having been missed in between. Their perihelion offsets were $\lesssim 0.5$ day, except for $\mathrm{P} /$ Gehrels 1 (orbital period $\approx 15$ years; recovered by Spacewatch) with $\Delta T=-4.4$ days. No comets expected at such apparitions were missed. SP comets missed at expected perihelia were: $\mathrm{P} /$ Neujmin 2, $\mathrm{P} /$ Denning-Fujikawa and $\mathrm{P} / \mathrm{de}$ Vico-Swift in $1987, \mathrm{P} / \mathrm{du}$ Toit in 1988, and P/Perrine-Mrkos in 1989. The last two comets were badly placed for observation. $\mathrm{P} /$ Neujmin 2 and $\mathrm{P} / \mathrm{de}$ Vico-Swift might have serious ephemeris uncertainties owing 
to intervening jovian perturbations since the last observations, $\sim 60$ and 20 years ago, respectively. P/Denning-Fujikawa seems to exhibit irregular activity because search failures showed it to be unexpectedly faint; its observational history is sporadic with observations only in 1881 and 1978, and it is on Kresák's (44.103.036) list of comets with past dormant phases. Large unmodelled or varying nongravitational effects may further complicate recovery of $\mathrm{P} /$ Neujmin 2, P/Perrine-Mrkos and P/Denning-Fujikawa.

Observations of comets at large distances from the Sun are important, both for astrometry in order to reach an improved description of the nongravitational effects (cf. III.2) and for photometry and imaging in order to monitor remote activity or deduce bulk properties of the nuclei. Such observations were done successfully, especially by Meech and her collaborators using large reflectors at Mauna Kea and Tololo. Thus the recent record set by the 1986 observations of comet Bowell (1982 I) at 13.6 AU from the Sun (Meech and Jewitt, 44.103.191) was broken when comet Černis (1983 XII) was observed at 15.3 AU on 6 April 1989 (Meech, MPC 16294). Other comets observed at more than $10 \mathrm{AU}$ during the past triennium include $\mathrm{P} / \mathrm{Halley}$ at $12.5 \mathrm{AU}$ (West, IAUC 5059) when most of the activity earlier reported (West and Jørgensen 50.103.151; West, $A B A$ 228, 531, 1990) had ceased, comet Shoemaker (1985 XII) at $12.7 \mathrm{AU}$ (Meech, $M P C$ 16295 ) and P/Neujmin 1 at $10.7 \mathrm{AU}$ (Meech, MPC 16294/5). Chiron was observed from 13 to $11 \mathrm{AU}$ inbound. Near-aphelion observations were made of P/Arend-Rigaux and P/Machholz, which are henceforth regarded as annual comets.

The Minor Planet Center issued a new edition of the magnetic tape file of observations containing 33672 observations of comets, complete to October 1989. Astrometric observations were provided and published in the $M P C$ by many observatories with some, as usual, being quite prolific. Particular mention is deserved by the material from Klet (A. Mrkos and Z. Vávrová; $\sim 400$ observations of LP comets and $>250$ of SP comets), Spacewatch at Kitt Peak (> 300 observations of SP comets until May 1988), Mauna Kea ( 250 observations of SP comets), and the Japanese amateur observatories (YGCO Hoshikawa, Nagano and Chiyoda, and Kitami; together $\sim 400$ observations of LP comets and 300 of SP comets). Details of observations made at Skalnaté Pleso were published by Pittich and Svoreñ (49.103.027, Contr.Astr.Obs.Sk.Pleso 19, $39,1990)$. Several other papers discuss ground-based astrometry of recent comets, in particular $\mathrm{P} /$ Halley. A very accurate position of the $\mathrm{P} /$ Halley nucleus was derived from Giotto data by Curdt et al. (45.103.407). Röser (44.103.436) compiled a catalogue of astrometric observations of $\mathrm{P} /$ Halley made during the 1909-1911 apparition. Astrometry of $\mathrm{P} /$ Halley was reviewed by West (49.103.108). More than 1100 observations were made in the USSR (Kiev Comet.Circ., Nos. $367-411)$.

\section{III.2 Orbits and Ephemerides; Orbital Evolution}

The orbital evolution of SP comets was reviewed by Carusi and Valsecchi (46.103.006). Reviews about chaotic motion were given by Froeschlé (46.042.016; ACM III 63). A 1989 workshop on the evolution of cometary orbits was summarized by Valsecchi ( $A C M$ III 615).

Orbital elements and ephemerides of comets by Marsden, Green, Nakano and others are routinely published in the IAU Circulars and Minor Planet Circulars. During recent years an attempt was made to give in a systematic manner the complete set of predicted orbital elements for all the returning periodic comets in a particular year, e.g. the elements for returns in 1993, were published in June 1990 MPCs. Cometary ephemerides are also prepared by Nakano and Green for an annual Comet Handbook (issued, essentially jointly, by the Oriental Astron. Assoc. and the International Comet Quarterly). From 1990, the ephemerides in Handbook Brit. Astron. Assoc. were extensively curtailed. Ephemerides for selected one-apparition comets to reappear during the period 1987-2000 were computed by Townsend and Rogers (44.103.054). The 6th edition of the CBAT/MPC Catalogue of Cometary Orbits was prepared by Marsden and published in December 1989 in both printed and diskette form. The 1292 entries represent a $9 \%$ increase over the 1986 edition and refer to 810 individual comets, 155 of them of short period. As compared with the 5 th edition, the number of SP comets with nongravitational parameters has increased by 11 , and there are 23 comets for which nongravitational solutions were extended to longer time intervals. After the catalogue appeared, nongravitational parameters were deter- 
mined for three more short-period comets. A new table in the catalogue gives the heliocentric osculating and the barycentric original and future values of the reciprocals of the semimajor axes for the 264 best-determined nearly-parabolic orbits. The more recent determinations of original and future orbits were presented by Marsden (Astron.J. 99, 1971, 1990) with particular emphasis on the evolution of the comet pair 1987 XXX - 1988 III. Although Radzievskij et al. (44.103.053) claimed the identity of comets $1770 \mathrm{II}$ and $1979 \mathrm{X}$ on the basis of similar elements and Tisserand parameters, this is the first well-defined pair of LP comets ever recorded. It is in all likelihood the result of a past splitting, and in this sense it is akin to the Kreutz group of sungrazing comets. Continuing earlier work on this group, Marsden (50.103.033) demonstrated that comets $1843 \mathrm{I}$ and $1880 \mathrm{I}$ in all probability separated from each other at their previous perihelion passage during the second half of the 15 th century. Their common parent may thus be linked to comet $1963 \mathrm{~V}$, suggesting a separation in late 1487 or less probably in mid-1463. It would be interesting if contemporary observational records of the appearance of a sungrazing comet could be found. New orbit computations were made for the six Solwind and ten SMM sungrazers. Indications are that all the $S M M$ sungrazers are closely related to the same subgroup as comets $1843 \mathrm{I}, 1880 \mathrm{I}$ and $1963 \mathrm{~V}$, and a case was also made for considering the Solwind comets to be members of this subgroup.

The nongravitational parameters referred to above are all derived using the "standard model" with constants $A_{1}$ and $A_{2}$ multiplying a function of heliocentric distance to describe the transverse and radial acceleration components in the orbital plane. In this framework, Forti (49.103.030) discussed the motions of several periodic comets and Szutowicz (44.103.201) concentrated on $\mathrm{P} /$ Wolf-Harrington. Rickman et al. (44.103.221) analyzed the nongravitational behaviour of $\mathrm{P} /$ Kopff when linking recent apparitions, attempting a physical interpretation of the results with the aid of a thermal model of the nucleus. However, there is a clear development toward more sophisticated models of the nongravitational force in future work. An obvious example is comet $\mathrm{P} / \mathrm{Halley}$, for which the abundance of recent accurate observations enables a closer look into the details of the motion including a center of light/center of mass offset (Chodas and Yeomans 45.103.504) possibly correlated with the brightness changes of the comet (Sarychev et al. 44.103.693). In his thesis, Landgraf (45.103.526) made a comprehensive analysis of these effects and in addition to the standard model considered an alternative deduced from the RickmanFroeschlé thermal models. Diaz-Bobillo and Zadunaisky (46.103.438) sought a further refinement of the modelling in terms of Gauss-Markov processes. For P/Halley Rickman (50.103.158) found that the nongravitational delay of 4 days is principally caused by the perihelion asymmetry of outgassing from the nucleus, causing the jet acceleration to be stronger after perihelion than before. Indications of a similar nature had come from a large sample of comets using light curves as indicators of such asymmetries (Rickman et al. 44.103.043). Important progress was made by Yeomans and Chodas (50.102.056) who introduced a simple perihelion asymmetry into the standard formula for nongravitational acceleration and thus found improved fits for several short-period comets by choosing the asymmetries in agreement with those of the light curves. As a result of this work, and of that by Festou et al. ( $A C M$ III 313), it seems that Bessel's conjecture back in 1836 , i.e. that the nongravitational effect stems from a perihelion asymmetry of a radial outflow from the nucleus, has now been confirmed. Festou et al. (Nature 345, 235, 1990) described how their correlation between nongravitational effects and light curve asymmetries was used to predict the large offset of the perihelion passage of $\mathrm{P} /$ Brorsen-Metcalf in 1989 (IAUC 4804).

Considering motion on a longer time-scale, Sitarski (44.103.171) introduced a new method of orbit improvement, using a combination of modern positional observations and the times of perihelion passage deduced from observations at older apparitions, enabling him to fit nongravitational parameters as smooth functions of time over two centuries for comet $\mathrm{P} /$ Encke. Application of this method to $\mathrm{P} / \mathrm{Halley}$, first with $A_{2}$ as a linear function of time (Sitarski and Ziolkowski 44.103.594) and then using a second order polynomial (Sitarski 46.103.530) showed that the fit to observed perihelion times in -239 is sensitive to the details of nongravitational modelling and that extrapolation with different models back to the 15th century BC results in differences of several decades between predicted perihelion times. For P/Encke, an extrapolation of the motion back to 1201 was attempted using a sinusoidal representation of the varying 
nongravitational effect that was found convenient for the interval 1786-1986 (Sitarski 46.103.143).

The orbital evolution of comet $\mathrm{P} /$ Halley was investigated over a range of longer time scales, using different models. Evidence from the structure of its meteor stream was discussed by Hajduk (44.104.072), Hajduková (44.104.097), and Jones et al. (49.104.040). While the former authors favour an age near 2300 revolutions, the latter prefer a shorter history spanning only 300 revolutions. Carusi et al. (46.103.532) discussed the results of an N-body integration back to $9367 \mathrm{BC}$ which shows a behaviour similar to, but not strictly verifying, the $\omega$ libration suggested by Kozai. Close encounters with Jupiter at the descending node seemed possible at the end of this integration. From the above-described results on the nongravitational behaviour it is obvious that nothing can be said about the actual occurrence or detailed outcome of such encounters. However, qualitative studies of the dynamics of comet Halley over a time scale of $\sim 10^{6}$ yrs by Froeschlé and Gonczi (46.102.058) and Dvorak and Kribbel $(A \& A$ 227, 264, 1990), by means of Lyapunov characteristic exponents and surfaces of section, respectively, clearly show a chaotic motion in simple dynamical models. A two-dimensional mapping was constructed by Chirikov and Vecheslavov $(45.103 .422,50.103 .194)$ to represent the motion of comet Halley, and the iteration of this mapping shows chaotic regions confined by regions of regular motion. The role of Saturnian perturbations in modifying the chaotic behaviour was discussed (also by Dvorak and Kribbel), and a weak nongravitational force was found to influence strongly the expected solar system lifetime of the comet. A completely stochastic approach was used by Olsson-Steel (44.103.595) to estimate this lifetime from encounter probabilities and ejection probabilities associated with encounters, and his result was 68000 orbits. Based on such probabilities, he subsequently (46.103.437) found that the likely capture route of comet Halley leads from a not too distant transneptunian region (see below) via a drastic perturbation by Neptune.

Chaos was identified by Petrosky and Broucke (46.042.048), and by Sagdeev and Zaslavskij (44.102.040), as a general feature for the motion of LP comets, using canonical mappings to represent the essential features of the dynamics. Strong dynamical chaos occurs for comets captured as temporary satellites of Jupiter (Murison 50.042.068). Rapid divergence of cometary heliocentric trajectories was found by Carusi et al. (45.103.021) and Todorovic-Juchniewicz (ACM III 459). Attention was also paid to resonant motions (Emel'yanenko 44.102.028, 46.102.051; Belyaev and Emel'yanenko, ACM III 253). Carusi et al. discovered high-order librations of Halley-type comets in direct orbits $(44.102 .050,46.103 .007)$, and Benest with co-workers found possible librations around the $1 / 1$ jovian resonance for P/Ge-Wang (Celest.Mech. 47, 361, 1990), the 5/3 resonance for $\mathrm{P} /$ Ciffré $(A \& A \mathbf{2 2 8}, 539,1990)$, and the $4 / 3$ resonance for $\mathrm{P} /$ Maury $(A C M$ III 255). A very peculiar orbital evolution was found for comet $\mathrm{P} / \mathrm{Machholz}$ (Green et al., Science 247, 1063, 1990) involving a large-scale oscillation of eccentricity and inclination on a period near 4000 yrs under the influence of the nearby $2 / 1$ resonance. The comet's perihelion thus moves between Earth's orbit and the solar corona.

\section{III.3 Cometary Origin, Dynamics and Spatial Distribution}

Cometary dynamics was reviewed by Rickman and Froeschlé (46.102.056) and by Marsden (44.102.043). In particular, the Oort cloud was reviewed by Weissman (Nature 344, 825, 1990) and by Scholl (44.102.042). A review of the relationships between comets and other minor bodies of the solar system was given by Kresák and Štohl (ACM III 379). The Origin of Comets by Bailey, Clube and Napier (Pergamon Press 1990) gives a historical as well as up-to-date review of related theories, hypotheses and arguments.

The Oort cloud, or a remote cometary reservoir in general, attracted widespread interest during the past triennium. To a large part, this development is a continuation of the advances made earlier during the 1980's in the dynamical theory of such reservoirs. The influence of the galactic tidal field on Oort cloud motion perpendicular to the galactic plane was included in many studies - e.g., by Duncan et al. (44.107.021) on the origin of the cloud (modelled as an outcome of planetesimal scattering from the Uranus-Neptune accretion zone) and its subsequent evolution. Earlier suggestions of a dense inner core to the cloud, extending outward from a few thousand $\mathrm{AU}$, were thus supported. According to Bailey and Stagg (46.102.021), the influx of comets from the inner core back into the Uranus-Neptune region yields a rich source of Jupiter 
family comets (and, probably, crater-forming impactors on the terrestrial planets) - perhaps too rich compared to observed fluxes, depending on the degree of central condensation. Stothers (45.102.005) argued that the lack of evidence for strong cratering episodes on Earth speaks against a strongly condensed inner core. Bailey (ACM III 221) and Bailey and Stagg (Icarus $\mathbf{8 6}, 2,1990$ ) claim that the cloud structure predicted by Duncan et al. is in a narrow range of possibilities set by the constraints of avoiding both overproduction of SP comets and inability to replenish an unstable outer Oort cloud.

However, it has been disputed whether the inner core with its nearly spherical symmetry is a viable source for the low-inclination Jupiter family. Duncan et al. (45.102.051) and Quinn et al. (Ap.J. 355, 667, 1990) made computer simulations of cometary capture indicating that a flattened source is needed, and they concluded that the transneptunian belt of icy planetesimals suggested long ago by Kuiper (the "Kuiper belt") would be the most likely source for most SP comets. The outstanding problem in such computations, however, remains a prohibitive demand of CPU time for an accurate treatment of the dynamics. Hence the approximations used, notably an increase of the masses of the giant planets, were criticized by Stagg and Bailey (50.102.040) who, however, introduced other approximations in their Monte Carlo simulations. The particular problems of adopting the stochastic mapping technique of such simulations to the complex dynamics of cometary capture were discussed by Froeschlé and Rickman (46.102.057, 50.042.104), and a test of the technique was described by Baille and Froeschlé ( $A \& A$ 234, 539, 1990). The infeed of comets from the transneptunian belt into Neptune-crossing orbits poses a special problem, to which different solutions were proposed. Slow chaos was reported for fictitious orbits started just beyond Neptune (Torbett 50.102.059; Torbett and Smoluchowski, Nature 345, 49, 1990), leading to a possibility of continuous infeed over the age of the solar system. From the macro-accretion of Uranus and Neptune, Ip (50.101.020) found that Earthsized objects could be expected in the transneptunian region, providing for efficient gravitational stirring of the comet disk. Stern (Celest.Mech. 47, 267, 1990) found that perturbations by stars and molecular clouds would impose moderate eccentricity and inclination to transneptunian orbits. An alternative possibility was considered by Duncan et al. (50.042.069) who devised a mapping to study the evolution of near-circular orbits between the planets and found long-term survival in the Uranus-Neptune zone to be possible, implying a slow diffusion into planet-crossing orbits until the present time.

Search projects aimed at detecting sizeable objects in the outer solar system were unsuccessful in finding transneptunian objects (Kowal 49.098.020; Luu and Jewitt 45.091.024). Jackson and Killen (46.102.010) investigated the possibility of detecting a transneptunian comet disk by its IR emission and concluded that it may not be swamped by the zodiacal dust emission. Aumann and Good ( $A$ p.J. 350, 408, 1990) found from IRAS data that $\mathrm{G}$ stars in the solar neighbourhood tend to be surrounded by cold clouds consistent with comet belts, and that there may be a similar emission source in the solar system at 100-150 AU. Other suggestions of comet disks around stars in the Galaxy were made on the basis of the sublimation of icy bodies during the red-giant stages of the stars (Matese et al. 50.112.064) considering the observations of dust production around red giants, and by Stern et al. (Nature 345, 305, 1990) considering observations of $\mathrm{H}_{2} \mathrm{O} / \mathrm{OH}$ outflows around such stars.

The effect of the galactic tide on Oort cloud evolution and the distribution of long-period cometary orbits was discussed in a number of papers. Antonov and Todriya (45.102.040) concluded that the cumulative perturbations by distant stars act much more strongly on cometary orbits than single close passages by stars or molecular clouds, and they found it likely that the disk shape of the Galaxy would be reflected in the orbital distribution. Delsemme (44.102.051) identified an avoidance of both high and low galactic latitudes by the observed aphelion directions, as predicted by the first-order theory of galactic tides. Matese and Whitman (50.102.061) gave an analytic treatment whose results were used in a Monte Carlo study of the infeed into observable orbits, again demonstrating good agreement between observed orbits and a steady-state infeed from a spherically symmetric Oort cloud. Chepurova and Shershkina (44.102.062, 50.102.012) simulated Oort cloud dynamics including both stars, molecular clouds and the general galactic tidal field. Yabushita (45.102.012) investigated the galactic tidal infeed by integrating orbits. Focussing on the observed LP comets he claimed discrepancies with the first-order theory 
(49.103.034, 49.102.019); this was disputed by Matese and Whitman, and by Byl (Astron.J. 99, $1632,1990)$. Yabushita (50.102.032) also looked into possible departures from steady-state infeed in the observed orbital distribution. One of several possible explanations for the nondetection of extrasolar comets advanced by McGlynn and Chapman (50.102.062) was indeed that the present flux of Oort cloud comets might be much higher than the long-term average. Past comet showers were considered by Hut et al. (44.081.025), and a $15 \mathrm{Myr}$ periodicity in the comet flux resulting from the variation of the vertical tide with distance from the galactic plane was discussed by Clube (44.102.066). Staniucha ( $A C M$ III 439) reviewed some recent work on the problem of Oort cloud survival and concluded that the energy transfer rate to cometary orbits had been underestimated by using the impulse approximation. Zheng (49.102.057) studied stellar perturbations, indicating the Oort cloud to be very unstable.

Cometary formation with emphasis on the planetesimal scattering picture was reviewed by Safronov (44.102.045). Napier (46.102.007) instead argued for pre-existing planetesimals in the molecular cloud out of which the solar system arose. Marochnik et al. (46.102.047) found high values when estimating the mass $\left(0.03 M_{\odot}\right)$ and angular momentum of the Oort cloud and therefore favoured cometary formation at several hundred AU at least. Eruptive origins for comets continue to be argued by some authors, notably Drobyshevski (46.103.497), Radzievskij and Tomanov (45.102.070), and Guliev (46.102.028). The latter papers considered transplutonian planets or their moons as cometary parent bodies, and transplutonian comet families were claimed to exist by Guliev and Dadashov (50.102.060). Guliev and Bajramov (45.103.007) identified two groups of Jupiter family comets on the basis of brightness behaviour, and Guliev (46.103.034) suggested that they have formed by captures and eruptions, respectively. The possible evolution of comets into apparently asteroidal bodies and the resulting cometary contribution to the population of Apollo-Amor asteroids was reviewed by Weissman et al. ( $A$ II 880), and the latter aspect was discussed in detail by Wetherill (46.098.021); Rickman (46.098.130) summarized a 1988 discussion of related questions. Andreev et al. (ACM III, 493) studied gravitational capture of extinct comets by the terrestrial planets, and Olsson-Steel (46.098.035) estimated the dynamical lifetimes of Jupiter-crossing asteroids, or extinct comets, by his stochastic scheme. Ziolkowski (46.098.016; ACM III 215) claimed the motion of (1221) Amor to have exhibited an unmodelled, possibly nongravitational effect in 1956.

\section{Satellites (J.-E. ARLOT) \\ IV.1 Observations for Astrometric Purposes}

\section{IV.1.1 PhOTOGRAPHIC OBSERVATIONS}

USNO (Washington D.C., USA): Pascu and Schmidt (Astron.J., 99, 1974, 1990) continued observations of the Galilean satellites, the satellites of Mars and S I - VIII with the 26-inch refractor in Washington D.C.. The satellites of Uranus and Neptune (N I) were observed (Walker and Harrington 45.101.072). Observatorio Nacional - LNA (Itajubá, Brazil): positions from 264 plates of the satellites of Uranus (1982 - 1985) with the $1.6 \mathrm{~m}$-reflector were published (Veiga et al. 44.101.017). 37 plates of the satellites of Saturn, 74 plates of N I, 2 plates of N II were obtained and will be reduced. Kiso Observatory (Japan): positions from 46 plates of J VI, VII, VIII and IX (1986 - 1990) with the $105 \mathrm{~cm}$ Schmidt were partially published (Nakamura et al. 45.099.029). ESO (La Silla, Chile) and CFH (Hawaii, USA): positions of N II from observations in 1982 and 1984 with the $1.5 \mathrm{~m}$-Danish reflector at ESO and in 1982 with the $3.6 \mathrm{~m}-\mathrm{CFH}$ reflector were published (Veillet and Bois 45.101.005); positions of the Lagrangian satellites of Tethys and Dione were similarly obtained in 1981 and 1985 (Oberti et al. 50.100.028). Bordeaux Observatory (France): photographic observations of the satellites of Saturn made in 1984 (Dourneau et al. 50.100.075). Astrometric observations of Titan and Iapetus are made in the FK5 system with the automatic meridian circle at each opposition since 1985 (60 for Titan and 25 for Iapetus until 1990); they have not yet been published. La Palma Observatory (Canary Islands, Spain): photographic positions of N I were obtained with the 1m-reflector (Taylor et al. A\&A 232, 565, 1990). USSR Observatories: Mars: In view of the importance of determining accurately the orbit of Phobos for the Phobos space project, an observational 
campaign was organized in 1988. More than ten observatories within the USSR participated and two expeditions were sent abroad; the observations were processed and transmitted to the Space Navigation Center and other institutions for improvement of the orbits. 856 positions of Phobos and 937 positions of Deimos (r.m.s. $=0.12$ arcsec) were obtained at Majdanak during 19 nights (July 23 - Oct. 4, 1988) by staff members of GAISH (Moscow) (Novikov 49.097.010; Bugaenko et al., Uzbek.Fiz.Nauk 1989-3, 531) and in 1986 by the Goloseevo expedition. Observations of Phobos were made in 1986 at Abastumani (Kiseleva and Chanturija 46.097.150) and long series of observations were also obtained at Pulkovo, Ordubad and other observatories. Jupiter: Observations of the Galilean satellites continued at Pulkovo with the 26 -inch refractor and the normal astrograph (Kiseleva 45.099.007); 131 plates of the satellites were taken. At Abastumani 64 plates were obtained and 36 positions were determined at the Nikolaev. Observations of these objects were also made in Kitab, Tashkent and Goloseevo; 143 positions determined at Nikolaev in 1983 -1985 were published (Voronenko and Gorel' 46.099.056), 95 further positions (1986-87) are in press. Observations at Abastumani (1983-84) and at Ordubad were published (Kiseleva et al. 45.041.023, Bobylev and Dement'eva 46.099.116). Saturn: Observations of the satellites of Saturn were made at Abastumani (89 plates), at Goloseevo, in Kazan (33 plates) and Tashkent. Results of observations in 1976 with the 26-inch refractor at Pulkovo, in 1983-85 with the zone astrograph in Nikolaev, and of S II - VIII in 1983-84 at Abastumani have appeared (Tolbin 46.100.067; Voronenko and Gorel' 46.099.056; Kiseleva et al. 45.041.023). Uranus and Neptune: At Abastumani 91 plates with U III and U IV, and 83 plates with Neptune and N I were taken.

\section{IV.1.2 CCD OBSERVATIONS:}

The use of CCD's has continued to increase. USNO (Washington D.C., USA): J XIV, S XII, S XIII, S XIV, U V and N II were observed with the 61-inch reflector at Flagstaff; positions of U V (1981 - 85) were published (Pascu et al. 43.101.025) and positions of N II were sent to JPL for the Voyager mission at Neptune. La Palma Observatory (Canary Islands, Spain): positions of N I were obtained with the $1 \mathrm{~m}$-telescope (Taylor et al. A\&A, 232, 565, 1990). Pic du Midi (France): during the 1988 Mars opposition, observations of Phobos and Deimos were made using the $1 \mathrm{~m}$ telescope and 813 positions were obtained (Colas and Arlot: in press).

\section{IV.1.3 PHOTOMETRIC OBSERVATIONS:}

Mutual events of the Galilean satellites were observed in 1985 (Froeschle et al. 45.099.001; Melillo 44.099.051; Arlot et al. 49.099.010; Arlot et al. 50.099.085; Arlot et al. A 6 A Supp.Ser. 82, 513, $1990)$ and the timing of jovian eclipses was studied by Loader (45.099.013). Observations were reported by Westphal (44.099.038). Mutual events of Pluto-Charon were observed by Vasundhara and Bhattacharyya (45.101.077) and by Blanco et al. (50.101.129). The occultation of 28 Sgr by Titan gave accurate astrometric results from observations at many observatories, cf. V.3.

\section{IV.1.4 OTHER OBSERVATIONS:}

37 observations were made on-board Phobos-2 on 21 Febr., 28 Febr. and 25 March 1989, at a range from 1100 to $180 \mathrm{~km}$. Processing of those made in February contributed to successful spacecraft trim maneuvers. As a result, Phobos-2 was inserted into a "quasi-satellite" orbit (relative to Phobos) and continued to stay in the closest vicinity of Phobos. Astrometric observations of Phobos and Deimos from Mariner 9 (1971-72) were published by Duxbury and Callahan (49.097.018). 56 speckle interferometric observations of Charon were made in 1984-85 by Beletic et al. (49.101.018). During the triennium, two compilations of observations were published: a catalogue of 5767 ground-based astrometric observations of the satellites of Mars made during the period.1877-1982 (Morley 49.002.012) and a catalogue of 51000 observations of S I - VIII made during the period 1874-1989 (Strugnell and Taylor: A EA Suppl.Ser. 83, 289, 1990).

\section{IV.2 Comparison of Observations with Theories - Determination of Elements}

\section{IV.2.1 SATEllites OF MARS:}

The Phobos space project actively encouraged studies of the motions of the Martian satellites. Subsequent improvement of the orbits was achieved by means of the new ground-based obser- 
vations in 1988 and also from previously unpublished observations by Pascu in 1969 and 1971 . The use of the many, high-precision observations resulted in greatly improved agreement between orbits determined from ground-based observations and from on-board observations, respectively (Shor $A C M$ III 175). The $1 \sigma$ uncertainty of the predicted positions of Phobos was reduced to $5 \mathrm{~km}$ (Kudryavtsev et al. Preprint GAISH N2, Moscow, 19, 1989). As mentioned above, the improved orbital elements for Phobos were used for trim maneuvers of Phobos-2 and for on-board observations of Phobos, which immediately proved the high accuracy of the Phobos ephemeris; the (O-C) values did not exceed $3 \mathrm{~km}$ (Kudryavtsev et al., ibid). On-board observations of Phobos combined with telemetry data made it possible to improve the orbit, to facilitate the spacecraft navigation and to determine Phobos' mass from the gravitational perturbations of the spacecraft. The uncertainty of the position of Phobos is now considered to be equal to $2-3 \mathrm{~km}$ (Kudryavtsev et al., ibid). The observational data have been analyzed by Shor (46.097.049), Ivanov et al. (46.097.049), Jones et al. (49.097.016), Sinclair (50.097.023), Jacobson et al. (50.097.054), Morley (49.002.012; AAS/GSFC Int. Symp. on Orbital Mechanics and Mission Design (Greenbelt), Paper 89-181, 1989) and Chapront-Touzé (A6A 235, 447, 1990), in order to determine the orbital elements of the satellites, various physical parameters of Mars, and the secular acceleration of Phobos. In addition, extensive analyses (some unpublished) have been carried out by the space mission engineers of Intercosmos/Glavcosmos, ESA/ESOC and $N A S A / J P L$ in support of the Phobos mission. There is agreement that a secular acceleration of Phobos exists: the well-determined value from the observations is $0.00124 \pm 0.00002 \mathrm{deg} \mathrm{yr}^{-2}$, which in fact is not too far from Sharpless' original (1945) value of 0.00188 . It is now clear that the uncertainty of the value of the acceleration generated by the disparity of various determinations was caused by a misinterpretation of the time-scale of some early observations from the Lick Observatory in a widely-used listing, and further confounded by an error in the time-scale of an early release of the Mariner 9 data.

\section{IV.2.2 Satellites of Saturn:}

The orbital elements of S I - VIII were improved by means of 14000 photographic observations (1967-83), emphasizing the need to use the best available theories for Hyperion and Iapetus (Taylor and Chen 46.100.001). An analysis of the orbits of Titan, Hyperion and Iapetus by numerical integration with a fit to micrometer observations during 1873-1923 was also made (Harper et al. 50.100.029). Photographic observations made in 1971 were used for improvements of the orbital elements of Iapetus and Hyperion (Hatanaka 45.100.013) and observations of the Satumian system made in 1975 were compared with the theories of motion (Tolbin 44.100.030). From an analysis of Voyager 1 and 2 data and ground-based observations obtained during the 1966 and 1980 ring plane crossings, a determination of the orbits and masses of S X (Janus) and S XI (Epimetheus) was made (Yoder et al. 50.100.085).

\section{IV.2.3 SATELlites OF URANUS:}

Numerous works appeared during the past triennium because of the Voyager encounter with Uranus. After the encounter, JPL published observations as well as various dynamical studies (44.003.001). Results on the masses of U I - V were also published by Anderson et al. (45.101.018). An analytical ephemeris of U I - V was deduced from Laskar's GUST86 theory (Laskar and Jacobson 44.101.017). A comparison of ground-based observations with theory was made for 1982-85 observations (Veiga et al. 44.101.017). Batrakov and Nikolskaya (50.101.053) published improved orbital parameters for U I - V from photographic observations in 1968-86; the observations are reproduced with r.m.s. values $\sim 0.2$ to 0.3 arcsec.

\section{IV.2.4 SATELltes of NePTUNe:}

Orbital elements were determined from observations during 1982-84 (Veillet and Bois 45.101.005). Ephemerides used by the Voyager project were based on orbits determined by Jacobson ( $A \& A$ 231, 241, 1990).

\section{IV.2.5 Satellite of Pluto:}

Orbital elements of Charon were determined from speckle interferometric observations in 1984 
and 1985 (Beletic et al. 49.101.045).

\section{IV.3 Theoretical Studies}

A review paper on the long-term evolution of the orbits of natural satellites was published by Duriez (46.091.087). Other studies of the evolution of natural planetary satellite systems were published by Peale (50.107.061) and Horedt (49.107.002).

\section{IV.3.1 SATELLITES OF MARS:}

Morley $(A B A, \mathbf{2 2 8}, 260,1990)$ improved Sinclair's analytical orbital model of the satellites to an accuracy of about $100 \mathrm{~m}$, in order to be compatible with the accuracy of the best spacecraft observations of the satellites $(\sim 1 \mathrm{~km})$. Chapront-Touzé $(46.097 .056 ; A \& A 235,447,1990)$ has developed a new semi-analytical theory ESAPHO of the motion of Phobos, which aims at an accuracy of about $1 \mathrm{~m}$, in order to be compatible with the high accuracy of the tracking data that had been expected from the Phobos-lander.

\section{IV.3.2 SATEllites of SATURN:}

Sinclair's theory of lapetus has been compared with Sinclair and Taylor's numerical integration and the theory of the motion of Iapetus has been improved (Harper et al. 45.100.009). A study of periodic orbits in the Enceladus-Dione system was made (Bevilacqua et al. ODNAO, 13). A theory of the motion of the Lagrangian satellites of Tethys and Dione was established by Oberti ( $A \& B A$ 228, 275, 1990). Message (50.100.092) describes computer-based techniques which were developed in the construction of the long-period perturbations of Hyperion's orbit. A general theory of the motion of the first eight satellites of Saturn by Duriez and Vienne is in press $(A B A)$.

\section{IV.3.3 Satellites of URANUS:}

Theoretical work continued during the past triennium, mainly because of the Voyager encounter with Uranus. A semi-analytical solution for the eccentricities and longitudes of the pericenters of U I - V was made (Lazzaro et al. 44.101.025) and Lazzaro (ODNAO, 39) studied the effects of solar perturbations on the motion of U I - V. A study of the origin of the chaotic behaviour in the Miranda-Umbriel 3:1 resonance was made by Henrard and Sato (Celest.Mech. 47, 391, 1990). Studies were also made of the tidal evolution of the satellites in three papers by Tittemore and Wisdom: I: the passage of Ariel and Umbriel through the 5:3 mean motion commensurability (45.101.074); II: an explanation of the high orbital inclination of Miranda (49.101.028); III: the evolution through the Miranda-Umbriel 3:1, Miranda-Ariel 5:3 and Ariel-Umbriel 2:1 mean motion commensurabilities (Icarus 85 394, 1990). The secular perturbations of the satellites were investigated by Malhotra et al. (50.101.052) and the role of secondary resonances in the orbital history of Miranda by Malhotra and Dermott (Icarus 85, 444, 1990).

\section{IV.3.4 SATELLITES OF NePTUNE:}

Several studies were made concerning the perturbation of the motion of Nereid (Vieira-Martins ODNAO, 59); Alfimova and Gerasimov 44.101.050). An analytic modelling of the motion of Nereid was made by Oberti ( $A \& A$, in press); the tidal evolution in the Neptune-Triton system was discussed by Chyba et al. (50.101.039).

\section{IV.4 Research on Ephemerides - Predictions of Phenomena}

Jacobson (46.101.025) prepared ephemerides of N I - II for the Voyager Neptune encounter. Post-encounter ephemerides of N I - II and $1989 \mathrm{~N} 1$ were published by Jacobson et al. (AIAA Paper 90-2881, 1990). The occurrence of mutual phenomena of Galilean satellites in 1991 has led to the publication of several predictions of these events (Aksnes and Franklin Icarus 84, 542, 1990; Arlot $A \cup A$, in press). It will be possible to use infrared observations of occultations of Io by other satellites to determine positions and intensities of the hot spots as Io's surface is progressively covered. Such observations were made by Goguen et al. (46.099.123) during the last series of mutual events and are proposed for the next series by Goguen (46.099.071), 
Sinton (46.099.075), Arlot (46.099.117) and Arlot and Rocher (50.099.027). Detailed positions of the volcanoes during the occultations of Io are given by Sinton et al. (Proc. of IJW meeting, Pasadena, Sept. 1989). Circumstances for Pluto-Charon mutual events in 1988 were published by Tholen et al. (44.101.038).

\section{IV.5 Dynamics of the Ring Systems}

Jupiter's rings have been described by Showalter et al. (43.099.058), eccentric features were studied in the $\mathrm{C}$ ring of Saturn by Porco and Nicholson (44.100.024) and regular structures are displayed in the Cassini Division by Flynn and Cuzzi (50.100.080). Eplee and Smith (43.100.013) explored the spokes in the B ring of Saturn. Cuzzi and Burns (45.100.014) proposed the existence of a belt of moonlets to explain a depletion of charged particles around Saturn's $F$ ring. Rosen and Lissauer (46.100.012) deduce the properties of the Saturnian rings from a bending wave excited by Titan. Lissauer et al. (46.100.064) give a bombardment history of the Saturn system.

Uranus' rings have been analyzed by comparing the Voyager 2 data with ground-based observations of occultations by French et al. (45.101.008) for the visible wavelenghts, by Holberg et al. (44.101.026) for the UV, and by Gresh et al. (49.101.031) for the radio data. A model of formation of the Uranus rings was proposed by Esposito and Colwell (49.101.029). Normal modes of oscillation in narrow rings were studied by Papaloizou and Lin (46.062.054) and for the Uranian rings by Longaretti (50.101.150).

Borderies and Longaretti (44.091.046) describe the dynamical behaviour of planetary rings in term of streamlines. Meyer-Vernet and Sicardy (43.091.009) analyze the torque exerted by a satellite on a disk at the Lindblad resonances, while Borderies et al. (50.091.015) study the confinement of sharp edges by shepherd satellites. As shown by Greenberg (46.091.010), the physical properties of the particles are a key problem of ring dynamics and Wiesel (44.091.018) shows that some fragmented, narrow ringlets may be explained by very inelastic collisions. Lin et al. (44.042.010) propose a model for the confinement of planetary arcs.

A numerical approach is used by Brophy et al. (Icarus 83, 133, 1990) to present the evolution of a narrow two-component ring with different size particles and by Petit and Hénon (44.091.041, 45.091.046) to study the mass segregation and the confinement mechanism. Simulations of colliding particle rings are made by Salo (43.042.057) and by Wisdom and Tremaine (45.091.023).

A continuing effort is being made to observe Neptune's ring-like arcs from the ground by means of stellar occultations. Observational constraints and theoretical models of Neptune's arcs have been reviewed by Brahic and Hubbard (49.101.058) and by Lissauer and Nicholson (Adv. Space Res., 10-1, 231, 1990).

\section{Occultations (L.H. WASSERMAN)}

\section{V.1 Identification of Upcoming Occultations}

Computerized searches for future occultations are being performed independently by three investigators. Wasserman, Bowell, and Millis at Lowell published predictions for $1988 / 1989$ (44.096.011) and 1990/1991 (Astron. J. 99, 723, 1990). These searches include all minor planets whose angular diameters are expected to reach 0.08 arcsec during a given search year and are run against the positions of approximately 340,000 individual stars in the SAO, AGK3, Perth 70, Pleiades Position and Lick Voyager catalogues. The most recent paper includes predictions for Triton and Titan, as well as the asteroids. Dunham has searched a similar set of asteroids against a composite catalogue containing the above catalogues plus the US Naval Observatory $\mathrm{XZ}$ Catalog and various other astrographic catalogues. His results are published in the Occultation Newsletter $(O N)$; an annual summary appears in the January issue of Sky and Telescope. A third search is performed by Goffin (46.098.132 and $O N$ ) using Dunham's star list against asteroids chosen by size rather than angular diameter. Local circumstances are computed by Carroll (44.096.014 and 46.096.016), while Boninsegna (44.096.015, 46.098.134, and $O N$ ) and Thirionet (46.098.131) distribute Goffin's predictions in Europe.

Mink and Klemola are searching photographic plates for occultations by Uranus, Neptune, Pluto, and satellites of Uranus and Neptune through 1999 (to be published in Astron.J.). Mink 
and Buie are searching the Space Telescope Guide Star Catalog for occultations by Uranus, Neptune, and Pluto; Mink and Stern are searching the same catalogue for occultations by (2060) Chiron. Searches ahead of Neptune and Uranus for stars which are bright in the infrared have been carried out by Nicholson et al. (45.096.001). Goffin has looked for mutual occultations of asteroids (49.098.149).

\title{
V.2 Prediction Refinement
}

Klemola continued to provide photographic astrometric positions from the Lick $0.51 \mathrm{~m}$ astrograph for use in last-minute refinement of selected occultation predictions. Lowell Observatory, Black Birch Observatory, and the Bordeaux transit circle (44.098.069) have also provided astrometric measurements. Shor (43.098.024) described the refinement technique used at ITA. Updates for selected events have been circulated via selected local coordinators in various parts of the world.

\section{V.3 Observations}

Five asteroids were observed with a sufficient number of chords that a meaningful diameter could be determined. Millis et al. reported results for (1) Ceres (44.098.086); (47) Aglaja (50.098.034), (324) Bamberga (50.098.088) and (4) Vesta (Bull.AAS. 21, 1247, 1989) while Dunham et al. (Astron.J. 89, 1636, 1990) published the results from the occultation of 1 Vul by (2) Pallas, which had a record number of 130 chords. As often happens, a larger number of occultations were observed, but with an insufficient number of chords to determine a diameter.

A number of occultations involving Uranus and its rings were reported: Millis et al. (22 April 1982) (43.101.015); French et al. (1 May 1982) (43.101.040); Elliot et al. published a report on an event which occurred on $25 \mathrm{March} 1983$ (44.101.022) and also on an extremely slow occultation which occurred near a stationary point during March 1987 (45.101.134). French et al. (45.101.008) have used data from Uranian ring occultations and Voyager observations to further constrain ring orbit models.

Another series of Galilean satellite mutual occultations and eclipses begins in 1991, cf. IV.4.

A rare series of mutual occultations and eclipses of Pluto and Charon began in late 1984 and will end in late 1990. Sawyer et al. (44.101.033) and Fink and DiSanti (45.101.001) used these events to separate the light from Pluto and Charon and thus obtain spectra of each object separately. Such individual spectra have led to the detection of water ice on Charon by Marcialis et al. (44.101.037) and Buie et al. (44.101.049). Tholen and Buie have used the timings of these events to determine the sizes of Pluto and Charon and the geometry of the system (45.101.124, 46.101.011, 46.101.054). Crude surface maps of Pluto have been generated by Marcialis (45.101.068), Binzel (46.101.053), and Buie and Tholen (49.101.044). Binzel has looked for hemispherical color differences on Pluto and Charon (46.101.115).

In July 1989, Saturn, its rings, and Titan all occulted the bright star $28 \mathrm{Sgr}$. First results for Titan were reported by Hubbard et al. and Sicardy et al. (Nature 343, 350 and 353, 1990) and by Forrest and Nicholson (M.N.R.A.S. 243, 10P, 1990). Preliminary results for Saturn and the rings were reported in various abstracts in Bull.AAS. 21.

Four observations of occultations by comets have been reported. Hu et al. (43.103.161), Eritsyan and Akhverdyan (43.103.608), and Wang et al. (45.103.524) claim detections of diminution of the starlight passing through the comet's coma, while Crawford and McNally (43.103.667) report a negative detection of two diffuse interstellar lines in absorption and the possible detection of another (unidentified) absorption line.

\author{
R.M.West \\ President of the Commission
}

